\title{
A COLLECTIVE CHALLENGE: OPEN SCIENCE FROM THE PERSPECTIVE OF SCIENCE EUROPE
}

\section{by Marie Timmermann}

Abstract: Open Science aims to enhance the quality of research by making research and its outputs openly available, reproducible and accessible. Science Europe, the association of major Research Funding Organisations and Research Performing Organisations, advocates data sharing as one of the core aspects of Open Science and promotes a more harmonised approach to data sharing policies. Good research data management is a prerequisite for Open Science and data management policies should be aligned as much as possible, while taking into account discipline-specific differences. Research data management is a broad and complex field with many actors involved. It needs collective efforts by all actors to work towards aligned policies that foster Open Science.

Keywords: Open Science; funders; research institutions; research data management

\section{EINE KOLLEKTIVE HERAUSFORDERUNG: OPEN SCIENCE AUS DER PERSPEKTIVE VON SCIENCE EUROPE}

Zusammenfassung: Open Science zielt darauf ab, die Qualität der Forschung zu verbessern, indem die Forschung und ihre Ergebnisse offen zugänglich, reproduzierbar und verfügbar gemacht werden. Science Europe, der Verband der großen Forschungsförderer und Forschungseinrichtungen, befürwortet den Datenaustausch als einen der Kernaspekte von Open Science und fördert einen stärker harmonisierten Ansatz für Richtlinien zum Datenaustausch. Gutes Forschungsdatenmanagement ist eine Grundvoraussetzung für Open Science und die Richtlinien für das Forschungsdatenmanagement sollten so weit wie möglich aufeinander abgestimmt werden, unter Berücksichtigung fachspezifischer Unterschiede. Das Forschungsdatenmanagement ist ein weites und komplexes Feld mit vielen beteiligten Akteurlnnen. Es bedarfgemeinsamer Anstrengungen aller Akteurlnnen, um auf einen abgestimmten Ansatz hinzuarbeiten, der Open Science fördert.

Schlagwörter: Open Science; Fördergeber; Forschungseinrichtungen; Forschungsdatenmanagement

DOI: https://doi.org/10.31263/voebm.v72i2.2831

(C) Marie Timmermann

Dieses Werk ist lizenziert unter einer

Creative-Commons-Lizenz Namensnennung 4.0 International 


\section{Contents}

1. Introduction

2. Data Sharing in the context of Open Science

3. Research Data Management - opportunities and challenges

4. Towards the International Alignment of Research Data Management

5. A Framework for Discipline-specific Research Data Management

6. Tackling the challenges collectively to advance Open Science

\section{Introduction}

Open Science aims to make research and its outputs as well as methods and software openly available. It can be defined as a series of practices that, under the right conditions, enhances the quality of research by making results reproducible and accessible. Sharing research data is one of the core aspects of Open Science. The development of Open Science causes numerous strategic, conceptual, operational, and technical challenges to the various scientific communities that undertake data-driven research. As researchers and research organisations collaborate on different levels, such as projects, programmes, and facilities, these challenges need to be tackled collectively by all actors involved.

Science Europe, the association of major Research Funding Organisations (RFOs) and Research Performing Organisations (RPOs) in Europe, has worked together over the last few years with other research stakeholders to promote a more harmonised approach to data sharing policies.

\section{Data Sharing in the context of Open Science}

Quality-assured research data are key building blocks of the research process and often generate impact that goes well beyond their initial purpose. The wider sharing and re-use of these data can help to maximise the value of the original investment as the data are indispensable in verifying research findings and highly valuable in terms of supporting new research. Research data should be permanently, publicly, and freely available for re-use, unless there are legitimate reasons (including discipline-specific or privacy-related ones) for delayed or restricted access. Science Europe fosters sharing and re-use of data as one of the key elements of Open Science and promotes data being FAIR 1 , i.e. findable, accessible, interoperable and re-usable. Science Europe therefore supports the European Open 
Science Cloud (EOSC) which has the potential to change the way research is done in the digital age. It can support the scientific communities in many ways: fostering the FAIR principles for research data, ensuring the recognition of researchers' data skills, addressing issues of access, copyright, and data subject privacy, allowing easier replicability of results, limiting data wastage, and contributing to clarification of the funding model for data generation and preservation.

\section{Research Data Management - opportunities and challenges}

Data sharing requires good research data management (RDM). There is currently a lot of variation in RDM policies. RDM requirements can be defined by e.g. funders, by universities and other research performing organisations or by journals, all of which can have different approaches on what they request from the researchers. Differences also can be found in the level of detail requested for DMPs, the format requested and at what point during the process (e.g. with grant proposal, upon grant allocation) DMPs have to be presented. Many RFOs, RPOs, and research communities have developed or are currently developing their own requirements and templates, especially for data management plans (DMP). This can cause confusion for researchers and is particularly problematic as many researchers get their funding from different sources and/or work in collaborative projects.

There is an urgent need to align RDM policies to provide more clarity and guidance for researchers. RDM in general, and DMPs in particular, should not be a bureaucratic burden but instead be a useful support when planning and conducting a research project.

Aligning RDM policies will also facilitate the work of RFOs and RPOs. Some organisations are currently unsure about the practical possibilities of RDM and the best way to implement respective policies. Other actors in the research community, such as data archives, scientific publishers, or research infrastructures will also benefit from a more harmonised approach as RDM practices will be more streamlined, predictable, and comparable.

\section{Towards the International Alignment of Research Data Management}

The Science Europe Practical Guide to the International Alignment of Research Data Management ${ }^{2}$ ('RDM Guide') provides common core require- 
ments for data management plans (DMPs). It provides organisations and communities with a common basis from which they can develop respective policies. The requirements should be considered minimum elements that can be supplemented with more specific ones in line with the needs and practices of the discipline or organisation in question. Researchers will benefit from aligned policies as they will not have to deal with different requirements when working with different organisations.

The RDM Guide also provides guidance for the selection of trustworthy repositories. As providing access to data is one of the pillars of sound, reproducible scientific research, an increasing number of RFOs, RPOs, and journals require researchers to deposit their research data in repositories. There is currently no generally accepted list of such repositories, whereas general registries of repositories list more than 2,000 of them. The number of repositories is constantly evolving, and their maturity and trustworthiness are difficult to assess. Identifying an appropriate repository can therefore be a challenging task for researchers, their organisations, and funders.

It is always advisable to refer to broadly recognised discipline-specific or certified repositories in the first place. In some fields exist disciplinespecific repositories for research outputs from a particular domain. These repositories have specific policies and standards in place that address the needs of the respective communities. They are not limited to a specific institution, but open to all researchers from a specific community. Repositories that serve a more general public have necessarily more generic policies and standards. A number of repositories have been certified by one of several acknowledged certification bodies, such as, but not limited to, the Core Trust Seal, the nestor Seal or other bodies based on DIN 31644 and ISO 16363:2012. To facilitate the recognition of trustworthy repositories for researchers, it is strongly recommended that repositories that have not yet been certified seek certification by such a body. Discipline-specific or certified repositories should always be the first choice. The criteria for the selection of trustworthy repositories presented by Science Europe are meant to support researchers or their organisations in cases where no such repository can be identified.

In recent years, diverse stakeholders from research funders to publishers have endorsed the FAIR Data Principles that enhance the re-use of data. The core requirements for DMPs and criteria for the selection of trustworthy repositories presented by Science Europe are compliant with the FAIR Data Principles, and even go beyond them on aspects such as storage and backup during the project and long- term preservation. Data 
management based on these core requirements and criteria will therefore support researchers in evaluating the level of 'FAIRness' of their data.

Since its publication in late 2018, a number of Science Europe member organisations, such as the Austrian $\mathrm{FWF}^{3}$, have integrated the RDM Guide in their organisational policies or are currently working on the implementation of its requirements and recommendations (e.g. in the Netherlands, Luxembourg, Ireland). In some countries such as Finland ${ }^{4}$ and Sweden ${ }^{5}$ the RDM Guide has been integrated into national RDM policies. Other countries such as France currently work on a national approach integrating the requirements of the RDM Guide. The European Commission refers to the Guide in its updated (June 2019) version of the Annotated Model Grant Agreement $^{6}$ for Horizon 2020.

\section{A Framework for Discipline-specific Research Data Management}

The amount, nature, and handling of data can differ quite significantly from one discipline to another. There cannot be one DMP template that is suitable for all disciplines. A discipline-specific approach is therefore needed. The so-called Domain Data Protocols (DDPs) can provide a pragmatic solution to guide researchers and ensure proper implementation of individual DMPs.

The Science Europe Guidance Document Presenting a Framework for Discipline-specific Research Data Management ${ }^{7}$ encourages and enables scientific communities to set up DDPs according to their specific needs. Individual researchers can then use the protocols as template for their DMPs in any given research field. This will support good research practice, ensure research integrity, and make RDM planning and evaluation easier for all parties involved. Researchers are provided with clear guidance for the development of their DMPs. RFOs, RPOs, and research communities can benefit from a systematic approach that makes it easier for them to assess, compare, and evaluate DMPs. Instead of having to evaluate and monitor many individual DMPs, funders and research organisations would simply require project proposers to comply with the relevant protocol. This would result in much shorter DMPs, reducing the time needed to review and evaluate them, as well as the time needed for researchers to create them. Several disciplines have already shown their interest in developing DDP templates for their researchers. Science Europe will monitor the developments and promote the uptake of the DDP templates through its members and contacts within the disciplines. In some countries, such as Portugal and 
the Netherlands, Science Europe member organisations or related data archives are in dialogue with different disciplines, supporting them through workshops in their development of their specific DDPs.

In the interest of aligning RDM policies as much as possible, disciplinespecific DMP requirements should be based on the DMP core requirements and developed to accommodate the specific needs of the field. They would pay special attention to standards and guidelines for data management that are relevant for a specific field or research community that shares similar data collection and processing methods. A DDP would not replace the responsibility of individual researchers to have and to work according to a DMP. Researchers should also not be required to blindly obey such a DDP (deviations from the protocol should be possible as long as an explanation is provided), but it will allow them to state that their DMP complies with the protocol of their field.

\section{Tackling the challenges collectively to advance Open Science}

For a successful alignment of RDM policies across countries and organisations, the concerned stakeholders need to work together. A number of RFOs have already implemented the DMP core requirements or are currently working on their implementation. Various RFOs work together with universities, research ministries, and other stakeholders in their respective countries in order to ensure a broad approach that would facilitate the work of researchers, their organisations, funders, and other involved actors. Other organisations that foster data sharing have already contacted Science Europe to identify ways to collaborate and promote the approach and several scientific communities have voiced their interest to work together to develop DDPs for their respective disciplines.

Research data management is a broad and complex field that involves many actors. It should be as aligned as possible and as discipline-specific as necessary. With a collective effort from all stakeholders involved RDM policies can be aligned to support research organisations, funders, and researchers in their work. Science Europe will keep promoting the uptake of the RDM Guide as well as the DDP templates. It will also further build on achievements so far to advocate further alignment of RDM to facilitate the work of researchers across Europe. The results of these efforts will also contribute to the development of the European Open Science Cloud (EOSC). As a member of the EOSC Executive Board Science Europe aims to ensure that the EOSC will be established in a way that it best suits researchers. 
1 Wilkinson, M. D. et al. (2016): The FAIR Guiding Principles for scientific datamanagement and stewardship. Scientific Data 3, 160018. https:// doi.org/10.1038/sdata.2016.18

2 Science Europe (2018): Practical Guide to the International Alignment of Research Data Management. https://www.scienceeurope.org/media/jezkhnoo/se_rdm_practical_guide_final.pdf

3 Austrian Science Fund (FWF), Research Data Management: https:// www.fwf.ac.at/en/research-funding/open-access-policy/research-data-management/

4 Academy of Finland, Data management plan: https://www.aka.fi/en/ funding/apply-for-funding/az-index-of-application-guidelines/datamanagement-plan/

5 Swedish Research Council, Data management plan: https://www.vr.se/ english/calls-and-decisions/grant-terms-and-conditions/data-management-plan.html

6 European Commission (2019): H2020 Programme. AGA - Annotated Model Grant Agreement, Version 5.2. http://ec.europa.eu/research/ participants/data/ref/h2020/grants_manual/amga/h2020-amga_ en.pdf

7 Science Europe (2018): Guidance Document. Presenting a Framework for Discipline-specific Research Data Management. https://www.scienceeurope.org/media/nsxdyvqn/se_guidance_document_rdmps.pdf 